Research Article

\title{
Revealing the Mechanism of Astragali Radix against Cancer- Related Fatigue by Network Pharmacology and Molecular Docking
}

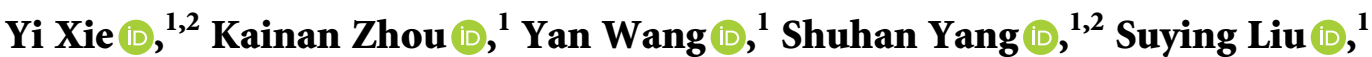 \\ Xueqian Wang $\mathbb{D}^{1}{ }^{1}$ and Ying Zhang $\mathbb{D}^{1}$ \\ ${ }^{1}$ Guang'anmen Hospital, China Academy of Chinese Medical Sciences, Beijing 100053, China \\ ${ }^{2}$ Beijing University of Chinese Medicine, Beijing 100029, China \\ Correspondence should be addressed to Ying Zhang; zylzy501@163.com
}

Received 25 September 2021; Accepted 22 November 2021; Published 8 December 2021

Academic Editor: Shagufta Perveen

Copyright $\odot 2021$ Yi Xie et al. This is an open access article distributed under the Creative Commons Attribution License, which permits unrestricted use, distribution, and reproduction in any medium, provided the original work is properly cited.

\begin{abstract}
Background. Cancer-related fatigue (CRF) is an increasingly appreciated complication in cancer patients, which severely impairs their quality of life for a long time. Astragali Radix (AR) is a safe and effective treatment to improve CRF, but the related mechanistic studies are still limited. Objective. To systematically analyze the mechanism of AR against CRF by network pharmacology. Methods. TCMSP was searched to obtain the active compounds and targets of AR. The active compound-target (AC-T) network was established and exhibited by related visualization software. The GeneCards database was searched to acquire $\mathrm{CRF}$ targets, and the intersection targets with AR targets were used to make the Venny diagram. The protein-protein interaction (PPI) network of intersection targets was established, and further, the therapeutic core targets were selected by topological parameters. The selected core targets were uploaded to Metascape for GO and KEGG analysis. Finally, AutoDock Vina and PyMOL were employed for molecular docking validation. Results. 16 active compounds of AR were obtained, such as quercetin, kaempferol, 7-O-methylisomucronulatol, formononetin, and isorhamnetin. 57 core targets were screened, such as AKT1, TP53, VEGFA, IL-6, and CASP3. KEGG analysis manifested that the core targets acted on various pathways, including 137 pathways such as TNF, IL-17, and the AGE-RAGE signaling pathway. Molecular docking demonstrated that active compounds docked well with the core targets. Conclusion. The mechanism of AR in treating CRF involves multiple targets and multiple pathways. The present study laid a theoretical foundation for the subsequent research and clinical application of AR and its extracts against CRF.
\end{abstract}

\section{Introduction}

Cancer-related fatigue (CRF) is a long-lasting physical, emotional, and/or cognitive tiredness or exhaustion associated with cancer or cancer treatment [1]. There is a huge distinction between CRF and other types of fatigue, which do not match the amount of recent activity, seriously interfere with body function, and even lead to the interruption of antitumor therapy. From 50\% to $90 \%$ of cancer patients suffer from CRF during treatment or even after the end of treatment [2]. The study result indicates that $30 \%-60 \%$ of cancer patients develop moderate to severe fatigue in therapy that leads to the discontinuation of oncological treatment. In addition, more than one-fourth of patients still experience fatigue for more than 5 years after successful treatment, which seriously reduces the quality of life [3]. The development of CRF is mainly associated with inflammation, immune dysregulation, hypothalamic-pituitary-adrenal axis disturbances, and reduced energy metabolism [4]. Since the specific pathogenesis of CRF has not been stated clearly, clinical drugs are still in the exploratory stage. The current interventions are mainly exercise and targeted psychological and mental-body treatments, but the therapeutic effect is still not ideal [5].

Astragali Radix (AR) is the dried root of Astragalus membranaceus Bunge. As an effective and widely accepted treatment, AR has been applied to a variety of diseases. AR was first contained in the ancient Chinese herbal book Shennong Ben Cao Jing in the Han Dynasty and is listed as a good product for tonifying qi and tonifying deficiency. It was 
demonstrated that AR has immunomodulatory, antioxidant, and anti-inflammatory effects by modern pharmacological studies [6].

AR has a significant effect on CRF. Clinical practice shows that traditional Chinese medicine decoction and injection with AR, the main drug, can effectively improve the symptoms of CRF and is widely used in the treatment. Jeong et al. used multiple evaluation scales to prove that Buzhong Yiqi decoction with $\mathrm{AR}$ as the main drug can effectively alleviate the fatigue state of cancer patients [7]. $\mathrm{Xu}$ et al. found that Renshen Yangrong decoction containing a large amount of AR can significantly reduce the fatigue level of patients with moderate-to-severe CRF [8]. In addition, a multicenter, double-blind, randomized phase IV study showed that Astragalus polysaccharide injection can be used as an effective and safe treatment to relieve CRF [9].

Network pharmacology can map herbal compounds and disease phenotypes into biomolecular networks and conduct a complex network of disease-gene-target-drug through qualitative and quantitative analysis [10, 11]. Molecular docking calculates the matching degree of binding between molecular ligands and protein receptors, which is able to validate the analysis results of network pharmacology [12].

Consequently, this study made an elaborate investigation into the pharmacological mechanism of AR in treating CRF by network pharmacology (Figure 1). It furnishes a theoretical basis for the further investigation and clinical practice of AR against CRF. Firstly, the compounds and corresponding targets of AR were searched through TCMSP, and CRF targets were searched through the GeneCards database. AR targets were intersected with CRF targets to acquire the Venny diagram. The AC-T network was framed through Cytoscape 3.8.2. The STING platform was utilized to establish the PPI network. Then, according to the topological parameters, the core targets were extracted. Metascape was utilized to proceed GO and KEGG analysis. Finally, the molecular docking of compound ligands and target receptors was verified through AutoDock Vina.

\section{Materials and Methods}

2.1. Gathering of Active Compounds and Prediction of Targets. "Huangqi" (AR) was used as a search term in TCMSP [13] (http://tcmspw.com/tcmsp.php) to acquire related compounds. The aforementioned compounds that conformed to the criterion were selected and regarded as active compounds of AR: oral bioavailability $(\mathrm{OB}) \geq 30 \%$ and drug likeness $(\mathrm{DL}) \geq 0.18$ [14]. The corresponding targets were extracted as AR targets. In order to prevent deviation caused by different naming methods, AR targets and subsequent CRF targets were normalized in the UniProt protein database [15] (https://www.uniprot.org).

2.2. Collection of CRF Targets. Using "cancer-related fatigue" as the keyword, the GeneCards database [16] (http://www. genecards.org) was searched to gain CRF targets. Through EXCEL software, the intersection of AR targets and CRF target was taken to draw a Venny diagram.
2.3. Establishment of AC-T Network. An AC-T network was established to display visually the intricate relationships between compounds and targets by the employment of Cytoscape 3.8.2 [17]. Network feature analysis was performed by the "Network Analyze" plugin to screen the main active compounds.

2.4. Establishment of PPI Network and Filtrating of Core Targets. The intersection targets were transmitted to the STRING platform [18] (https://string-db.org) to obtain PPI relationships. The CSV file containing the PPI relationship was downloaded to establish the PPI network. In this network, degree centrality (DC) determined the color depth and diameter size of the node. Targets with DC exceeding the average value were screened as core targets.

2.5. GO and KEGG Analysis. As an online tool for gene enrichment analysis, Metascape [19] (http://metascape.org/) integrated more than 40 gene function annotation databases. The core targets were uploaded into the Metascape platform to carry on Go and KEGG analysis. Data with $P<0.05$ were selected as the analysis results.

2.6. Molecular Docking. Searching PDB database (http:// www.rcsb.org) and TCMSP, structural files of the main active compounds as well as core targets were downloaded and saved as pdbqt format. The coordinate files of receptors and ligands were preprocessed by AutoDockTools 1.5.6 software and saved in pdbqt format: the water molecules in the ligands were removed, the ligands and receptors were separated, nonpolar hydrogen was added, and the Gasteiger charge was calculated. Through the gradient algorithm and multithreading technology of AutoDock Vina software, the docking activity between receptors and ligands can be evaluated by molecular docking score. Docking scores $<-4.25,<-5.0$, and $<-7.0$, respectively, represent the existence, good, and strong docking activity between the ligand and the receptor. The docking of molecular ligands and protein receptors was intuitively exhibited through PyMOL software.

\section{Results}

3.1. Compounds of $A R$ and Intersection Targets. 87 compounds of AR were extracted through TCMSP. 16 active compounds (Table 1) were selected through $\mathrm{OB}$ and $\mathrm{DL}$ parameters. Targets corresponding to active compounds were extracted and transmitted into EXCEL software, the repeated items were deleted, and a total of 199 AR targets were acquired after merging.

1620 CRF targets were collected through the GeneCards database. AR targets and CRF targets were mapped, resulting in a total of 131 intersection targets. The mapping result of AR targets and CRF targets was made into the Venny diagram (Figure 2). 


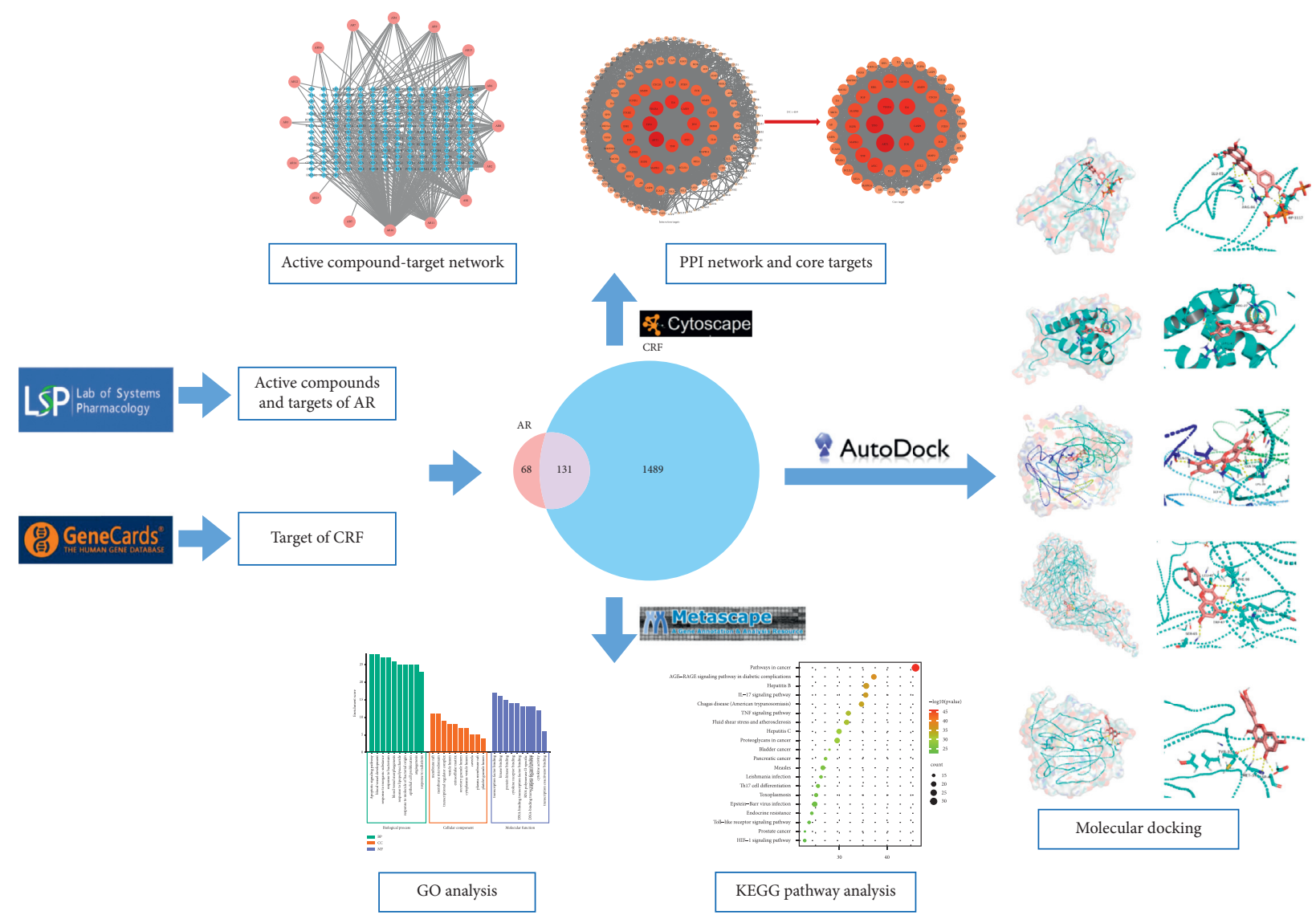

FIGURE 1: Workflow of the present research.

TABle 1: Active compounds of AR.

\begin{tabular}{|c|c|c|c|c|}
\hline Number & Molecule ID & Molecule name & $\begin{array}{l}\mathrm{OB} \\
(\%)\end{array}$ & DL \\
\hline AR1 & MOL000378 & 7-O-Methylisomucronulatol & 74.69 & 0.3 \\
\hline AR2 & MOL000392 & Formononetin & 69.67 & 0.21 \\
\hline AR3 & MOL000433 & FA & 68.96 & 0.71 \\
\hline AR4 & MOL000380 & $(6 \mathrm{aR}, 11 \mathrm{aR})-9,10-$ Dimethoxy-6a, 11a-dihydro-6H-benzofurano $[3,2-\mathrm{c}]$ chromen-3-ol & 64.26 & 0.42 \\
\hline AR5 & MOL000211 & Mairin & 55.38 & 0.78 \\
\hline AR6 & MOL000371 & 3, 9-Di-O-methylnissolin & 53.74 & 0.48 \\
\hline AR7 & MOL000239 & Jaranol & 50.83 & 0.29 \\
\hline AR8 & MOL000354 & Isorhamnetin & 49.6 & 0.31 \\
\hline AR9 & MOL000417 & Calycosin & 47.75 & 0.24 \\
\hline AR10 & MOL000098 & Quercetin & 46.43 & 0.28 \\
\hline AR11 & MOL000422 & Kaempferol & 41.88 & 0.24 \\
\hline AR12 & MOL000442 & 1, 7-Dihydroxy-3, 9-dimethoxy pterocarpene & 39.05 & 0.48 \\
\hline AR13 & MOL000296 & Hederagenin & 36.91 & 0.75 \\
\hline AR14 & MOL000379 & 9, 10-Dimethoxypterocarpan-3-O- $\beta$-D-glucoside & 36.74 & 0.92 \\
\hline AR15 & MOL000033 & $\begin{array}{l}\text { (3S,8S,9S,10R,13R,14S,17R)-10,13-Dimethyl-17-[(2R,5S)-5-propan-2-yloctan-2-yl]- } \\
2,3,4,7,8,9,11,12,14,15,16,17 \text {-dodecahydro-1H-cyclopenta[a]phenanthren-3-ol }\end{array}$ & 36.23 & 0.78 \\
\hline AR16 & MOL000387 & Bifendate & 31.1 & 0.67 \\
\hline
\end{tabular}

3.2. AC-T Network. In the AC-T network (Figure 3), the DC of a single target indicates the number of connected nodes. The network was analyzed by the "Network Analyze" plugin, and the active compounds were ranked by DC. The top five active compounds (Table 2) were set out with betweenness centrality (BC), closeness centrality (CC), and DC.
3.3. PPINetwork and Core Targets. There were 131 nodes and 2676 edges in the PPI network of intersection targets. Node size and color depth were proportional to DC. Through further analysis of the topological parameters, nodes meeting the criteria of DC $\geq$ average value (40.9) were extracted. 57 core targets were screened out. The PPI 


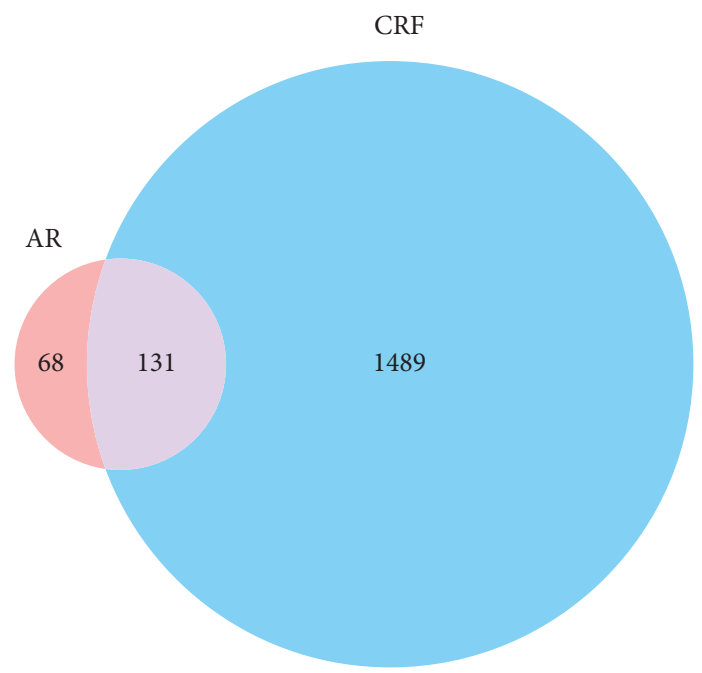

FIGURE 2: Venny diagram of AR targets and CRF targets (the area of the circle is proportional to the number of targets).

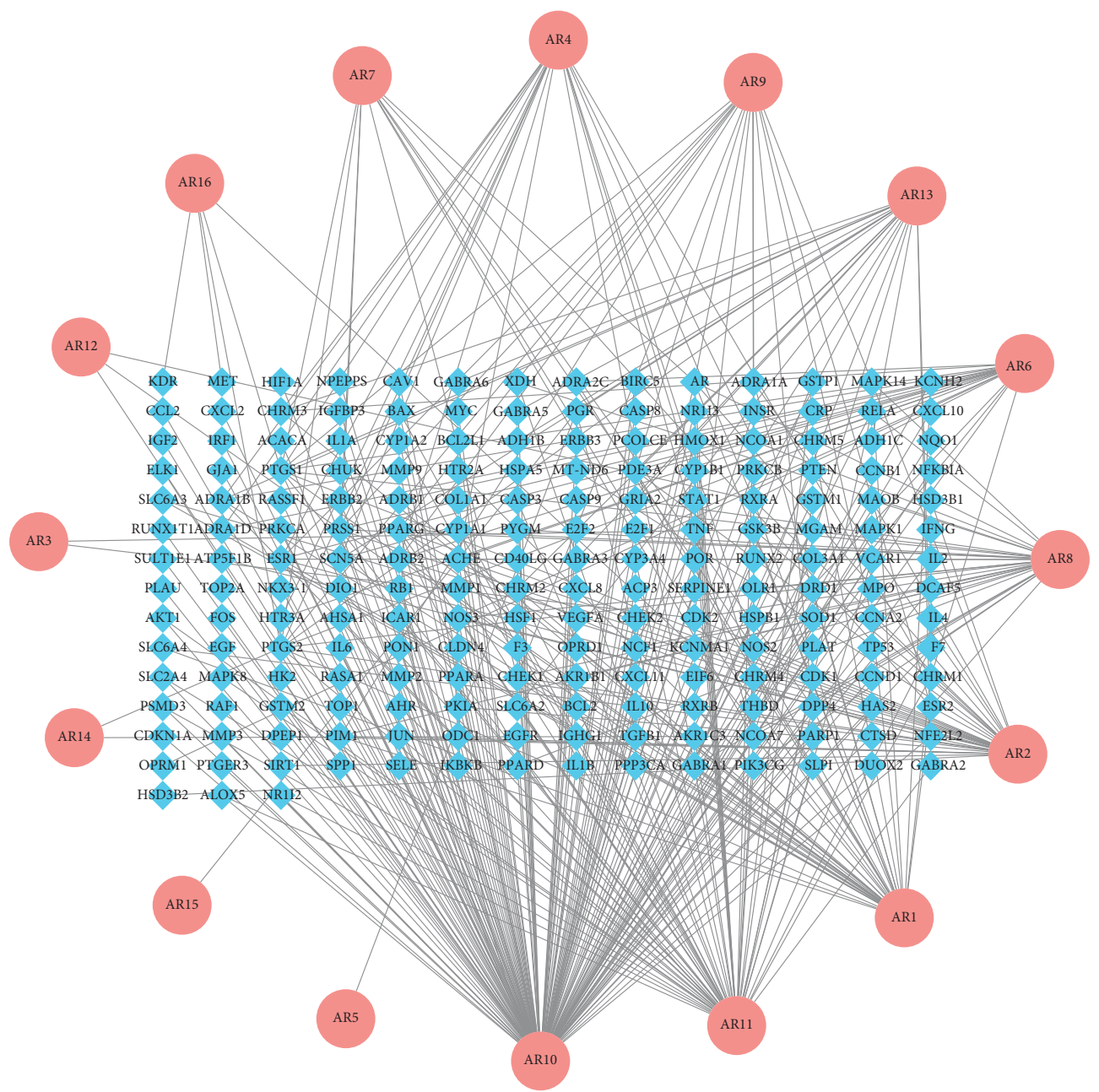

Figure 3: AC-T network.

network of intersection targets and core targets is displayed in Figure 4 . Ranked by DC, the top fifteen core targets are listed in Table 3.
3.4. GO and KEGG Analysis. The GO and KEGG analysis results of 57 core targets after the arithmetic by Metascape are shown in Figures 5 and 6. 
TABLE 2: The top five active compounds of AR.

\begin{tabular}{lcccc}
\hline Number & Molecule name & BC & CC & DC \\
\hline AR10 & Quercetin & 0.745 & 0.613 & 144 \\
AR11 & Kaempferol & 0.157 & 0.414 & 57 \\
AR1 & 7-O-Methylisomucronulatol & 0.119 & 0.388 & 40 \\
AR2 & Formononetin & 0.106 & 0.380 & 35 \\
AR8 & Isorhamnetin & 0.064 & 0.376 & 31 \\
\hline
\end{tabular}
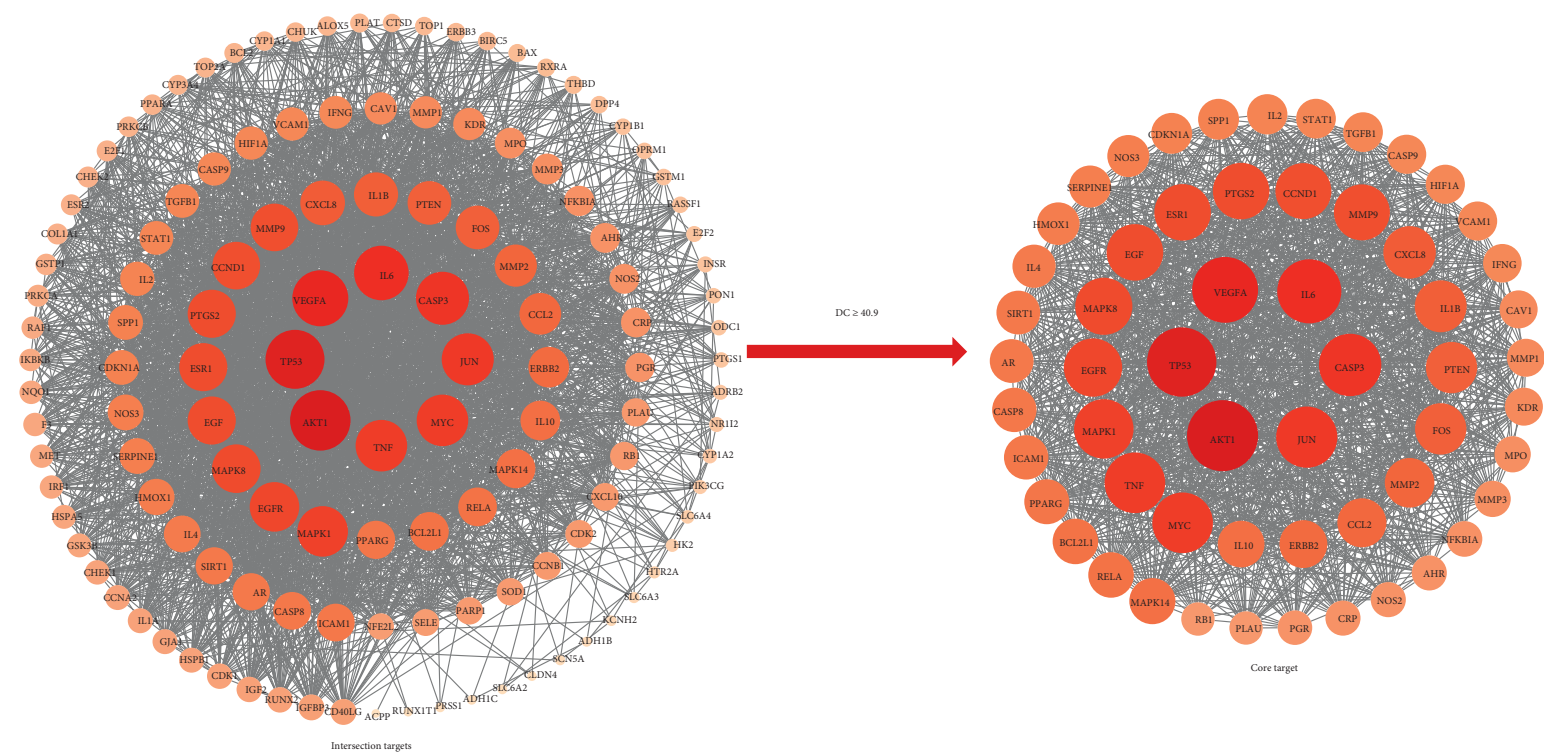

Figure 4: The PPI network of intersection targets and core targets (the larger the node, the darker the color, indicating the larger the DC).

TABle 3: The top fifteen core targets.

\begin{tabular}{|c|c|c|c|c|}
\hline Number & Gene name & $\mathrm{BC}$ & $\mathrm{CC}$ & DC \\
\hline 1 & AKT1 & 0.056 & 0.844 & 106 \\
\hline 2 & TP53 & 0.049 & 0.823 & 103 \\
\hline 3 & VEGFA & 0.029 & 0.788 & 97 \\
\hline 4 & IL-6 & 0.031 & 0.778 & 94 \\
\hline 5 & CASP3 & 0.019 & 0.756 & 91 \\
\hline 6 & JUN & 0.017 & 0.756 & 89 \\
\hline 7 & TNF & 0.020 & 0.747 & 88 \\
\hline 8 & MYC & 0.020 & 0.751 & 88 \\
\hline 9 & MAPK1 & 0.024 & 0.743 & 86 \\
\hline 10 & EGFR & 0.023 & 0.739 & 84 \\
\hline 11 & МАРК8 & 0.014 & 0.730 & 82 \\
\hline 12 & EGF & 0.022 & 0.722 & 81 \\
\hline 13 & PTGS2 & 0.014 & 0.722 & 81 \\
\hline 14 & ESR1 & 0.023 & 0.722 & 81 \\
\hline 15 & CCND1 & 0.013 & 0.710 & 80 \\
\hline
\end{tabular}

GO analysis $(P<0.05)$ manifested that 1552 entries of biological processes were yielded, such as the apoptotic signaling pathway, blood vessel development, and response to inorganic substances. 34 entries of cell components were yielded, such as membrane raft, membrane microdomain, and transcription regulator complex. 94 entries of molecular functions were yielded, such as transcription factor binding, kinase binding, and protein kinase binding.

KEGG analysis $(P<0.05)$ showed that AR treatment of CRF involved 137 pathways. The top 5 were pathways in cancer, AGE-RAGE pathway, hepatitis B, IL-17 pathway, and Chagas disease.
3.5. Molecular Docking. The top five active compounds were selected to dock to the top five core targets in turn to calculate the docking scores (Table 4 ).

All docking scores were $<-5.0$, and the abovementioned 5 active compounds docked well with the 5 core targets. Take quercetin as an example to show the visual pictures of its docking with 5 core targets, as shown in Figures 7-11.

\section{Discussion}

Assessment of AC-T network demonstrated that the top five active compounds in the treatment of CRF by $A R$ were 


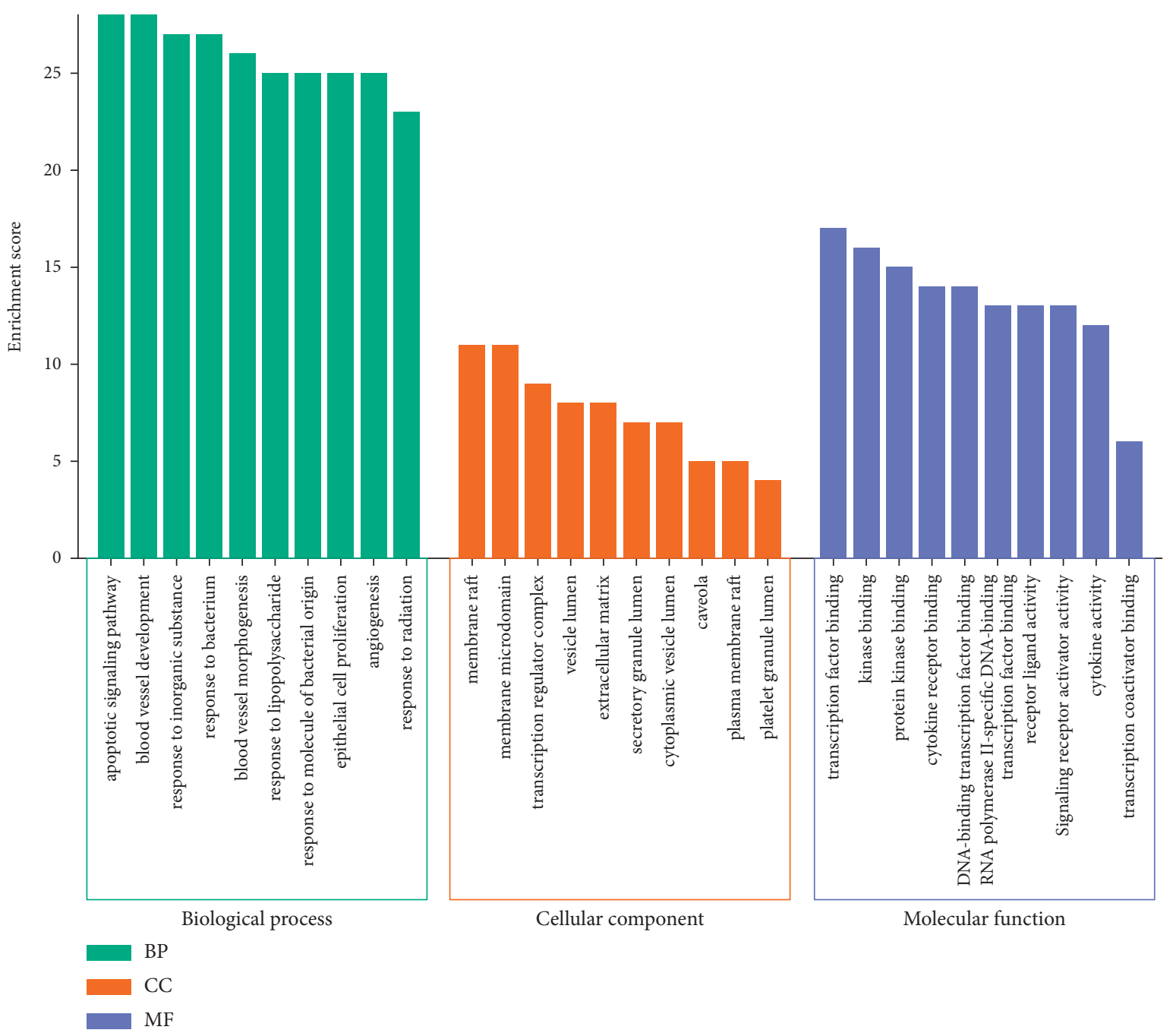

Figure 5: GO analysis of core targets.

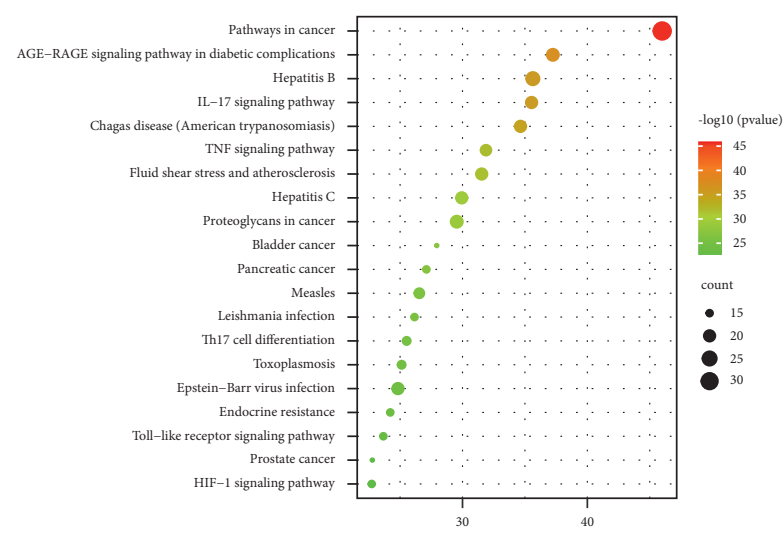

FIGURE 6: KRGG analysis of core targets.

quercetin, kaempferol, 7-O-methylisomucronulatol, formononetin, and isorhamnetin.

Quercetin exerts its antifatigue effect mainly by eliminating metabolic accumulation, and as an effective antioxidant, it has the ability to scavenge free radicals while regulating mitochondrial biogenesis [20]. Studies have
TABLE 4: Docking score.

\begin{tabular}{lccccc}
\hline & AKT1 & TP53 & VEGFA & IL-6 & CASP3 \\
\hline Quercetin & -5.9 & -5.7 & -7.5 & -8.0 & -7.5 \\
Kaempferol & -5.9 & -6.2 & -7.6 & -7.9 & -7.8 \\
7-O- & -5.6 & -6.1 & -6.8 & -6.6 & -6.8 \\
Methylisomucronulatol & -6.5 & -6.6 & -7.3 & -7.8 & -7.9 \\
Formononetin & -5.8 & -5.9 & -7.6 & -8.0 & -7.5 \\
Isorhamnetin & & & & &
\end{tabular}

shown that quercetin can change the energy metabolism of mice and effectively improve their physical endurance [21]. Quercetin can also exert antitumor activity by inducing apoptosis, inhibiting cell proliferation, and inhibiting angiogenesis and metastasis [22]. In addition, quercetin has pharmacological effects such as anti-inflammatory, antibacterial, antiviral, and immune regulation [23]. Many previous studies have proved that kaempferol has good antiinflammatory, immune regulation, antioxidation, antitumor, and other pharmacological properties [20]. It has been shown that kaempferol can induce Th1-mediated immune responses through dendritic cells [24]. Kaempferol can also 

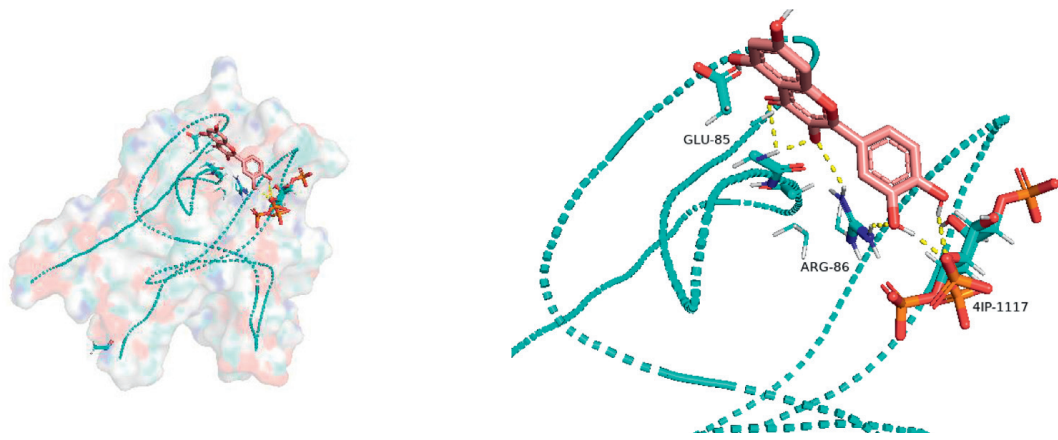

FIgURE 7: Docking of quercetin and AKT1.
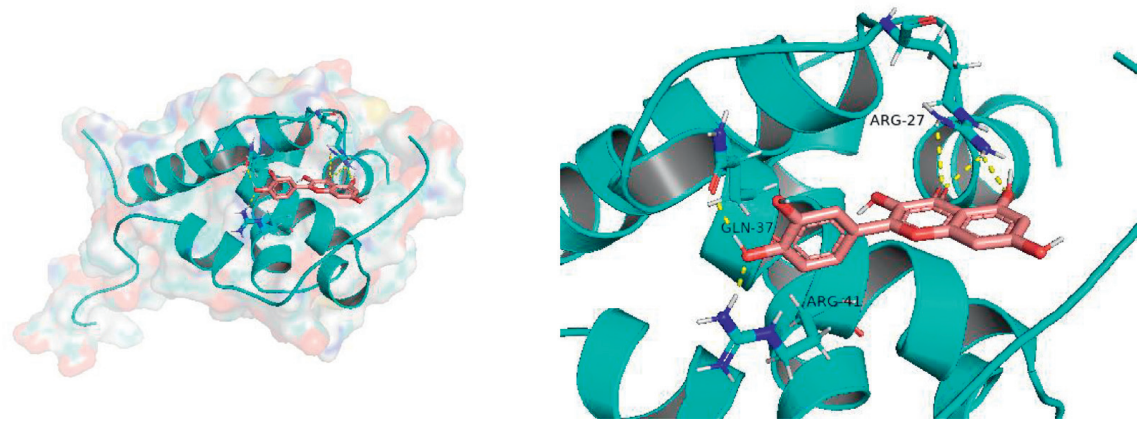

FIgURE 8: Docking of quercetin and TP53.
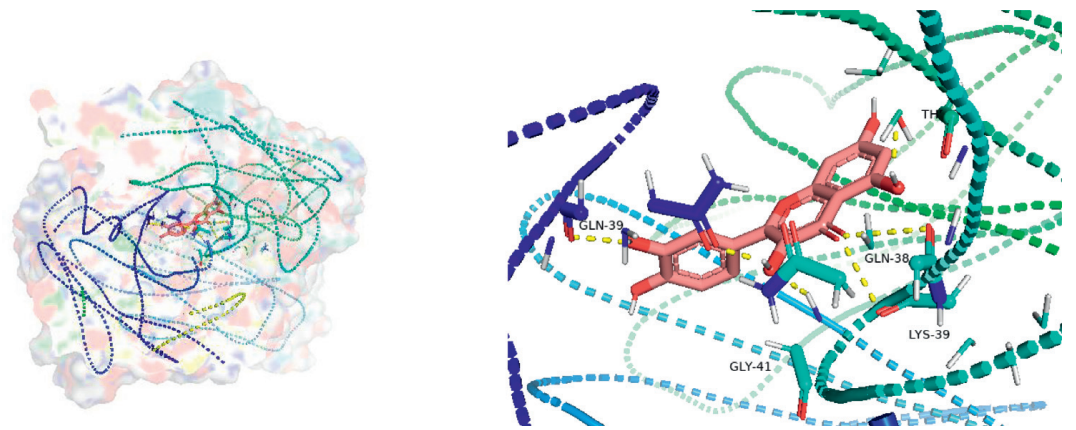

Figure 9: Docking of quercetin and VEGFA.
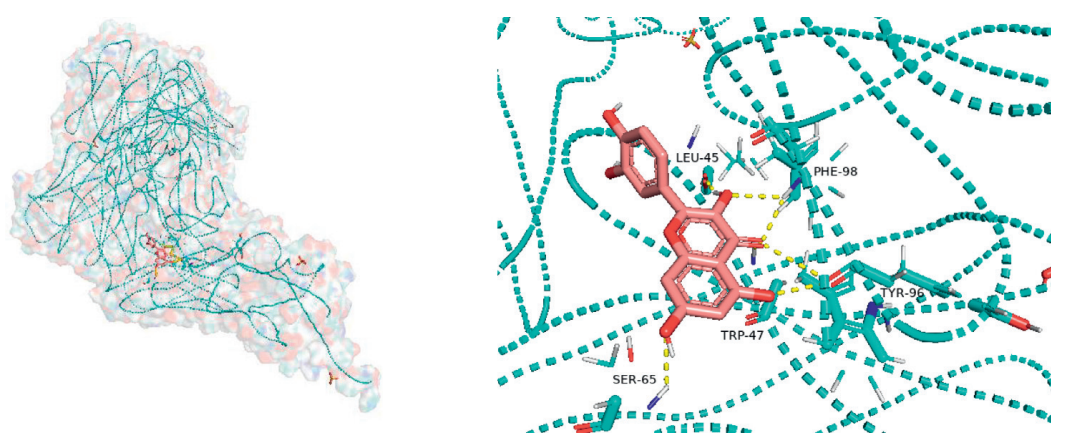

FIGURE 10: Docking of quercetin and IL-6.

exhibit antioxidant activities by increasing the expression of related antioxidant enzymes and reducing oxidative stress [25]. 7-O-Methylismucronulatol is a unique active component of $\mathrm{AR}$ and related research is rare at present. Formononetin is able to scavenge $\mathrm{DPPH}$ radicals in vitro and can promote the release of IL- 4 to achieve the purpose of improving fatigue [26]. In addition, formononetin can also regulate $\mathrm{Bcl}-2$, caspase-3, and other signaling pathways, inhibit tumor cell proliferation, and prevent tumor cell invasion [27]. Isorhamnetin is a powerful antioxidant, and 

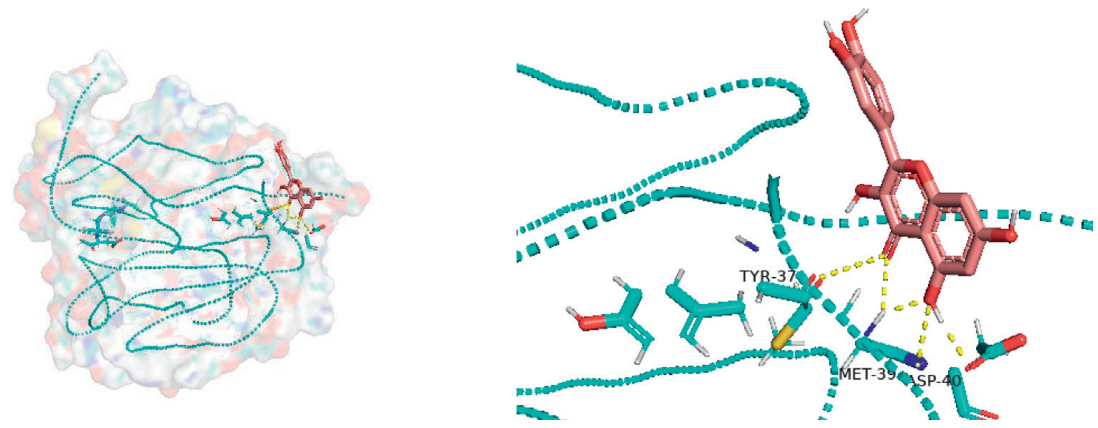

FIGURE 11: Docking of quercetin and CASP3.

its antioxidant effect also contributes to downregulating the expression of COX-2 in inflammatory responses [28]. It has been shown that isorhamnetin reduces the production of IL12 and TNF- $\alpha$ and elevates heme oxygenase 1 protein levels to exert anti-inflammatory properties $[29,30]$. Furthermore, isorhamnetin has antitumor effects and is extensively used in treating various tumors [31-33].

According to the attribute parameters of the core targets, AR mainly treats CRF through AKT1, TP53, VEGFA, IL-6, CASP3, and other targets.

AKT1 is one of the important isoforms of AKT. As a key signal downstream of PI3K, AKT mainly promotes cell growth, cell proliferation, and cell motility through the phosphorylation of a series of substrates [34]. The absence of AKT1 increases energy expenditure [35]. Liu et al. found that by upregulating the expression of the AKT1 gene, it can enhance the body's ability and exert anti-fatigue effects [36]. TP53 is the most commonly mutated gene in human cancer. The mutated TP53 is able to interfere with the recruitment and activity of T cells and myeloid cells and can lead to the loss of the cancer-suppressive function of wildtype TP53 [37]. TP53 controls inflammatory responses and regulates immunity and metabolism by regulating a variety of downstream target genes or molecules [38]. VEGFA is a key regulator of vascular growth. Landi et al. found that circulating levels of VEGFA were substantially decreased in patients with prolonged fatigue and that VEGFA was able to promote immunomodulators into the blood-brain barrier $[39,40]$. In addition, VEGFA is an important target for cancer therapy, and VEGF inhibitors such as bevacizumab are now widely used. IL-6 plays a significant part in inflammation and immune responses. Inagaki et al. found that increased fatigue in cancer patients was associated with increased IL-6 levels [41]. IL-6 can induce T helper cell activation and keep the balance of regulatory T cells and Th17 cells [42]. Moreover, IL-6 may lead to abnormalities in the hypothalamic-pituitary-adrenal axis [43]. CASP3 is the main executor of apoptosis and is associated with DNA fragmentation, chromatin condensation, and apoptosome formation. Existing studies have shown that CASP3 gene polymorphisms are associated with cancer risk [44].

KEGG analysis of core targets showed that AR treatment of CRF involved a variety of signaling pathways related to tumors: pathways in cancer, AGE-RAGE, IL-17, TNF, Tolllike receptor, and HIF-1 pathways.
In the AGE-RAGE signaling pathway, AGEs interact through AGE-RAGE to bind and trigger RAGEs that play a key role in inflammation and oxidative stress [45]. Furthermore, AGE-RAGE acts synergistically to affect programmed cell death signaling and promote cancer development [46]. In the IL-17 signaling pathway, IL-17 is a pleiotropic proinflammatory cytokine involved in various processes such as host defense, inflammatory diseases, tissue repair, and cancer development [47]. Studies have shown that IL-17 may promote cancer development by promoting chronic tissue inflammation and can prevent cancer cells from receiving immune surveillance [48]. In the TNF signaling pathway, as one of the important inflammatory mediators, TNF- $\alpha$ regulates immunity, host defense, and inflammation by regulating cytokine production, cell survival, and cell death [49]. Moss et al. found that in patients with fatigue, TNF- $\alpha$ dramatically multiplied [50]. In addition, present studies have proved that TNF- $\alpha$ is involved in regulating and interfering with energy metabolism [51]. In the Toll-like receptor signaling pathway, activation of TLRs may underlie the pathophysiology of multiple diseases including chronic fatigue syndrome, autoimmune diseases, and depression [52]. TLRs participate in the inchoate activation of immune response by recognizing host-derived macromolecules [53]. In addition, in different tumor types, the expression of TLRs may lead to tumor progression or regression [54]. In the HIF-1 signaling pathway, HIF-1 has the ability to regulate the related genes of energy metabolism and is involved in cellular accommodation to low oxygen [55]. Furthermore, the HIF-1 signaling pathway participates in key processes in various tumor biologies such as cell proliferation and apoptosis, immune response, and invasion and metastasis [56].

\section{Conclusion}

In summary, quercetin, kaempferol, 7-O-methylisomucronulatol, formononetin, isorhamnetin, and other active compounds in AR may exert positive effects via AKT1, TP53, VEGFA, IL6, CASP3, and other targets, and through AGE-RAGE, IL-17, TNF, Toll-like receptor, and HIF-1 signaling pathways in treating CRF.

\section{Abbreviations}

AC-T: Active compound-target 


\author{
AR: Astragali Radix \\ BC: Betweenness centrality \\ CC: Closeness centrality \\ CRF: Cancer-related fatigue \\ DC: Degree centrality \\ DL: Drug likeness \\ OB: Oral bioavailability \\ PPI: Protein-protein interaction.
}

\section{Data Availability}

The data used to support this research can be obtained from the corresponding author upon reasonable request.

\section{Conflicts of Interest}

The authors proclaim no conflicts of interest in this study.

\section{Authors' Contributions}

Yi Xie, Kainan Zhou, and Yan Wang conceived the study; Shuhan Yang and Suying Liu searched the relevant literature; Yi Xie and Xueqian Wang wrote the manuscript and produced diagrams; and Ying Zhang revised the manuscript. All authors approved the final manuscript.

\section{Acknowledgments}

This project was supported by Beijing Natural Science Foundation (no. 7192181) and "One Belt and One Road" Cooperation Project of China Academy of Chinese Medical Sciences in 2019 (no. GH201921).

\section{References}

[1] A. M. Berger, A. P. Abernethy, A. Atkinson et al., "NCCN clinical practice guidelines cancer-related fatigue," Journal of the National Comprehensive Cancer Network, vol. 8, no. 8, pp. 904-931, 2010.

[2] M. P. O. Campos, B. J. Hassan, R. Riechelmann, and A. Del Giglio, "Cancer-related fatigue: a practical review," Annals of Oncology, vol. 22, no. 6, pp. 1273-1279, 2011.

[3] J. E. Bower, "Cancer-related fatigue-mechanisms, risk factors, and treatments," Nature Reviews Clinical Oncology, vol. 11, no. 10, pp. 597-609, 2014.

[4] M. S. Y. Thong, C. J. F. Van Noorden, K. Steindorf, and V. Arndt, "Cancer-related fatigue: causes and current treatment options," Current Treatment Options in Oncology, vol. 21, no. 2, p. 17, 2020.

[5] A. Fabi, R. Bhargava, S. Fatigoni et al., "Cancer-related fatigue: ESMO clinical practice guidelines for diagnosis and treatment," Annals of Oncology, vol. 31, no. 6, pp. 713-723, 2020.

[6] Z. Guo, Y. Lou, M. Kong, Q. Luo, Z. Liu, and J. Wu, "A systematic review of phytochemistry, pharmacology and pharmacokinetics on astragali radix: implications for astragali radix as a personalized medicine," International Journal of Molecular Sciences, vol. 20, no. 6, p. 1463, 2019.

[7] J. S. Jeong, B. H. Ryu, J. S. Kim, J. W. Park, W. C. Choi, and S. W. Yoon, "Bojungikki-tang for cancer-related fatigue: a pilot randomized clinical trial," Integrative Cancer Therapies, vol. 9, no. 4, pp. 331-338, 2010.
[8] Y. Xu, X. S. Wang, Y. Chen, Q. Shi, T. H. Chen, and P. Li, “A phase II randomized controlled trial of renshen Yangrong tang herbal extract granules for fatigue reduction in cancer survivors," Journal of Pain and Symptom Management, vol. 59, no. 5, pp. 966-973, 2020.

[9] C.-H. Wang, C.-Y. Lin, J.-S. Chen et al., "Karnofsky performance status as A predictive factor for cancer-related fatigue treatment with Astragalus Polysaccharides (PG2) injection-A double blind, multi-center, randomized phase IV study," Cancers, vol. 11, no. 2, p. 128, 2019.

[10] T. T. Ho, Q. T. Tran, and C. L. Chai, "The polypharmacology of natural products," Future Medicinal Chemistry, vol. 10, no. 11, pp. 1361-1368, 2018.

[11] S. Li and B. Zhang, "Traditional Chinese medicine network pharmacology: theory, methodology and application," Chinese Journal of Natural Medicines, vol. 11, no. 2, pp. 110-120, 2013.

[12] L. Pinzi and G. Rastelli, "Molecular docking: shifting paradigms in drug discovery," International Journal of Molecular Sciences, vol. 20, no. 18, p. 4331, 2019.

[13] J. Ru, P. Li, J. Wang et al., "TCMSP: a database of systems pharmacology for drug discovery from herbal medicines," Journal of Cheminformatics, vol. 6, no. 1, p. 13, 2014.

[14] X. Xu, W. Zhang, C. Huang et al., "A novel chemometric method for the prediction of human oral bioavailability," International Journal of Molecular Sciences, vol. 13, no. 6, pp. 6964-6982, 2012.

[15] R. Apweiler, A. Bairoch, C. H. Wu et al., "UniProt: the universal protein knowledgebase," Nucleic Acids Research, vol. 32, pp. D115-D119, 2004.

[16] M. Safran, I. Dalah, J. Alexander et al., "GeneCards version 3: the human gene integrator," Database, vol. 2010, Article ID baq020, 2010.

[17] P. Shannon, A. Markiel, O. Ozier et al., "Cytoscape: a software environment for integrated models of biomolecular interaction networks," Genome Research, vol. 13, no. 11, pp. 2498-2504, 2003.

[18] D. Szklarczyk, A. L. Gable, D. Lyon et al., "STRING v11: protein-protein association networks with increased coverage, supporting functional discovery in genome-wide experimental datasets," Nucleic Acids Research, vol. 47, no. D1, pp. D607-D613, 2019.

[19] Y. Zhou, B. Zhou, L. Pache et al., "Metascape provides a biologist-oriented resource for the analysis of systems-level datasets," Nature Communications, vol. 10, no. 1, p. 1523, 2019.

[20] C. Luo, X. Xu, X. Wei et al., "Natural medicines for the treatment of fatigue: bioactive components, pharmacology, and mechanisms," Pharmacological Research, vol. 148, Article ID 104409, 2019.

[21] J. Wu, W. Gao, J. Wei, J. Yang, L. Pu, and C. Guo, "Quercetin alters energy metabolism in swimming mice," Applied Physiology Nutrition and Metabolism, vol. 37, no. 5, pp. 912-922, 2012.

[22] D. Kashyap, S. Mittal, K. Sak, P. Singhal, and H. S. Tuli, "Molecular mechanisms of action of quercetin in cancer: recent advances," Tumor Biology, vol. 37, no. 10, pp. 12927-12939, 2016.

[23] A. Massi, O. Bortolini, D. Ragno et al., "Research progress in the modification of quercetin leading to anticancer agents," Molecules, vol. 22, no. 8, p. 1270, 2017.

[24] J. Jeon, B.-C. Lee, D. Kim, D. Cho, and T. Kim, "Hydrophilic astragalin galactoside induces $\mathrm{T}$ helper type 1-mediated immune responses via dendritic cells," International Journal of Molecular Sciences, vol. 19, no. 10, p. 3120, 2018. 
[25] J. M. Calderon-Montano, E. Burgos-Moron, C. Perez-Guerrero, and M. Lopez-Lazaro, "A review on the dietary flavonoid kaempferol," Mini Reviews in Medicinal Chemistry, vol. 11, no. 4, pp. 298-344, 2011.

[26] Y.-H. Kuo, W.-J. Tsai, S.-H. Loke, T.-S. Wu, and W.-F. Chiou, "Astragalus membranaceus flavonoids (AMF) ameliorate chronic fatigue syndrome induced by food intake restriction plus forced swimming," Journal of Ethnopharmacology, vol. 122, no. 1, pp. 28-34, 2009.

[27] K.-C. Tay, L. T.-H. Tan, C. K. Chan et al., "Formononetin: a review of its anticancer potentials and mechanisms," Frontiers in Pharmacology, vol. 10, p. 820, 2019.

[28] K. Seo, J. H. Yang, S. C. Kim, S. K. Ku, S. H. Ki, and S. M. Shin, "The antioxidant effects of isorhamnetin contribute to inhibit COX-2 expression in response to inflammation: a potential role of HO-1," Inflammation, vol. 37, no. 3, pp. 712-722, 2014

[29] H. N. Jnawali, D. Jeon, M.-C. Jeong et al., "Antituberculosis activity of a naturally occurring flavonoid, isorhamnetin," Journal of Natural Products, vol. 79, no. 4, pp. 961-969, 2016.

[30] C. Boesch-Saadatmandi, A. Loboda, A. E. Wagner et al., "Effect of quercetin and its metabolites isorhamnetin and quercetin-3-glucuronide on inflammatory gene expression: role of miR-155i," The Journal of Nutritional Biochemistry, vol. 22, no. 3, pp. 293-299, 2011.

[31] S. M. Saud, M. R. Young, Y. L. Jones-Hall et al., "Chemopreventive activity of plant flavonoid isorhamnetin in colorectal cancer is mediated by oncogenic src and $\beta$-catenin," Cancer Research, vol. 73, no. 17, pp. 5473-5484, 2013.

[32] J. Hu, Y. Zhang, X. Jiang et al., "ROS-mediated activation and mitochondrial translocation of CaMKII contributes to Drp1dependent mitochondrial fission and apoptosis in triplenegative breast cancer cells by isorhamnetin and chloroquine," Journal of Experimental \& Clinical Cancer Research, vol. 38, no. 1, p. 225, 2019.

[33] K. A. Manu, M. K. Shanmugam, L. Ramachandran et al., "Isorhamnetin augments the anti-tumor effect of capeciatbine through the negative regulation of NF- $\kappa \mathrm{B}$ signaling cascade in gastric cancer," Cancer Letters, vol. 363, no. 1, pp. 28-36, 2015.

[34] I. Vivanco and C. L. Sawyers, "The phosphatidylinositol 3Kinase-AKT pathway in human cancer," Nature Reviews Cancer, vol. 2, no. 7, pp. 489-501, 2002.

[35] M. Wan, R. M. Easton, C. E. Gleason et al., "Loss of Akt1 in mice increases energy expenditure and protects against dietinduced obesity," Molecular and Cellular Biology, vol. 32, no. 1, pp. 96-106, 2012.

[36] F. X. Liu, Z. X. Lin, H. L. Zhang et al., "Analysis of anti-fatigue mechanism and potential targets of ginseng," Zhongguo Zhongyao Zazhi, vol. 44, no. 24, pp. 5479-5487, 2019.

[37] J. Blagih, M. D. Buck, and K. H. Vousden, "p53, cancer and the immune response," Journal of Cell Science, vol. 133, no. 5, Article ID jcs237453, 2020.

[38] T.-X. Lu, K. H. Young, W. Xu, and J.-Y. Li, “TP53 dysfunction in diffuse large B-cell lymphoma," Critical Reviews in Oncology, vol. 97, pp. 47-55, 2016.

[39] A. Landi, D. Broadhurst, S. D. Vernon, D. L. J. Tyrrell, and M. Houghton, "Reductions in circulating levels of IL-16, IL-7 and VEGF-A in myalgic encephalomyelitis/chronic fatigue syndrome," Cytokine, vol. 78, pp. 27-36, 2016.

[40] D. H. Dobrogowska, A. S. Lossinsky, M. Tarnawski, and A. W. Vorbrodt, "Increased blood-brain barrier permeability and endothelial abnormalities induced by vascular endothelial growth factor," Journal of Neurocytology, vol. 27, no. 3, pp. 163-173, 1998.
[41] M. Inagaki, M. Isono, T. Okuyama et al., "Plasma interleukin6 and fatigue in terminally ill cancer patients," Journal of Pain and Symptom Management, vol. 35, no. 2, pp. 153-161, 2008.

[42] M. F. Neurath and S. Finotto, "IL-6 signaling in autoimmunity, chronic inflammation and inflammation-associated cancer," Cytokine \& Growth Factor Reviews, vol. 22, no. 2, pp. 83-89, 2011.

[43] A. Kolak, M. Kamińska, E. Wysokińska et al., "The problem of fatigue in patients suffering from neoplastic disease," Współczesna Onkologia, vol. 2, no. 2, pp. 131-135, 2017.

[44] S. Yan, Y. Z. Li, X. W. Zhu, C. L. Liu, P. Wang, and Y. L. Liu, "HuGE systematic review and meta-analysis demonstrate association of CASP-3 and CASP-7 genetic polymorphisms with cancer risk," Genetics and Molecular Research, vol. 12, no. 2, pp. 1561-1573, 2013.

[45] C.-Y. Shen, C.-H. Lu, C.-H. Wu et al., "The development of maillard reaction, and advanced glycation end product (AGE)-receptor for AGE (RAGE) signaling inhibitors as novel therapeutic strategies for patients with AGE-related diseases," Molecules, vol. 25, no. 23, p. 5591, 2020.

[46] B. N. Waghela, F. U. Vaidya, K. Ranjan, A. S. Chhipa, B. S. Tiwari, and C. Pathak, "AGE-RAGE synergy influences programmed cell death signaling to promote cancer," Molecular and Cellular Biochemistry, vol. 476, no. 2, pp. 585-598, 2021.

[47] X. Li, R. Bechara, J. Zhao, M. J. McGeachy, and S. L. Gaffen, "IL-17 receptor-based signaling and implications for disease," Nature Immunology, vol. 20, no. 12, pp. 1594-1602, 2019.

[48] M. J. McGeachy, D. J. Cua, and S. L. Gaffen, "The IL-17 family of cytokines in health and disease," Immunity, vol. 50, no. 4, pp. 892-906, 2019.

[49] F. Balkwill, "TNF- $\alpha$ in promotion and progression of cancer," Cancer and Metastasis Reviews, vol. 25, no. 3, pp. 409-416, 2006.

[50] R. B. Moss, A. Mercandetti, and A. Vojdani, "TNF-alpha and chronic fatigue syndrome," Journal of Clinical Immunology, vol. 19, no. 5, pp. 314-316, 1999.

[51] X. Chen, K. Xun, L. Chen, and Y. Wang, "TNF- $\alpha$, a potent lipid metabolism regulator," Cell Biochemistry and Function, vol. 27, no. 7, pp. 407-416, 2009.

[52] K. Lucas and M. Maes, "Role of the toll like receptor (TLR) radical cycle in chronic inflammation: possible treatments targeting the TLR4 pathway," Molecular Neurobiology, vol. 48, no. 1, pp. 190-204, 2013.

[53] P. J. Maglione, N. Simchoni, and C. Cunningham-Rundles, "Toll-like receptor signaling in primary immune deficiencies," Annals of the New York Academy of Sciences, vol. 1356, no. 1, pp. 1-21, 2015.

[54] M. Dajon, K. Iribarren, and I. Cremer, "Toll-like receptor stimulation in cancer: a pro-and anti-tumor double-edged sword," Immunobiology, vol. 222, no. 1, pp. 89-100, 2017.

[55] C. Y. M. Okumura, A. Hollands, D. N. Tran et al., "A new pharmacological agent (AKB-4924) stabilizes hypoxia inducible factor-1 (HIF-1) and increases skin innate defenses against bacterial infection," Journal of Molecular Medicine, vol. 90, no. 9, pp. 1079-1089, 2012.

[56] L. Schito and G. L. Semenza, "Hypoxia-inducible factors: master regulators of cancer progression," Trends in Cancer, vol. 2, no. 12, pp. 758-770, 2016. 